\title{
TEM and Magnetic Studies of Metallic Nanoparticles in Ni-ion-implanted Rutile
}

\author{
X.T. Zu, ${ }^{*}$ S. Zhu, ${ }^{* *}$ X. Xiang, ${ }^{*}$ L.M. Wang ${ }^{* *}$ \\ * Department of Applied Physics, University of Electronic Science and Technology of China, \\ Chengdu, 610054, P.R. China \\ ** Department of Nuclear Engineering and Radiological Sciences, University of Michigan, Ann \\ Arbor, MI 48109-2104, U.S.A.
}

Ion implantation is one of the most powerful techniques to fabricate metal nanoparticles in the near surface of insulating substrates. Titanium dioxide $\left(\mathrm{TiO}_{2}\right)$ has attracted much attention because it is one of the wide band gap insulators and one of the most promising photocatalysts with high activity. Surface modification of $\mathrm{TiO}_{2}$ by $\mathrm{Cu}$ or $\mathrm{Ag}$ negative ion implantation can improve photocatalytic efficiencies by approximately 1.8 times [1]. Cr ion implantation has enhanced electrical conductivity of $\mathrm{TiO}_{2}$ single crystals [2]. Room temperature ferromagnetic properties of Co-doped $\mathrm{TiO}_{2}$ have been reported. [3]. Nano-sized Au particles in $\mathrm{TiO}_{2}$ have been synthesized by ion implantation for unusual nonlinear optical properties enhanced by surface plasmon resonance [4]. In our study, the magnetic nanoparticles of $\mathrm{Ni}$ embedded in $\mathrm{TiO}_{2}$ single crystals have been synthesized by ion implantation, which may provide potential application of the nanocomposite as magneto-optical materials for high density magnetic data storage device.

Nanoparticles of $\mathrm{Ni}$ in the near surface region of $\mathrm{TiO}_{2}$ single crystals have been synthesized by ion beam implantation. The Ni ion implantation was conducted at the room temperature to a fluence of $1 \times 10^{17} / \mathrm{cm}^{2}$. Transmission electron microscopy and a MPMS superconducting quantum interference device (SQUID) magnetometer have been utilized to characterize the nanostructure of Ni particles in $\mathrm{TiO}_{2}$, and the change of optical and magnetic properties. Nanopartilce of Ni with size ranging $3-18$ $\mathrm{nm}$ was observed in the surface of $\mathrm{TiO}_{2}$ and these nanoparticles have a specific orientation relationship with the matrix: $\mathrm{Ni}[011]_{\mathrm{Ni}} / /[010]_{\mathrm{TiO} 2}$. Magnetic measurement indicated that the coercive force of Ni nanoparticles was about $210 \mathrm{Oe}$ at $10 \mathrm{~K}$. The blocking temperature based on ZFC/FC curves was about $85 \mathrm{~K}$.

\section{Reference}

[1] H. Tsuji et al., Surface Coating and Technology 158-159 (2002) 208.

[2] R.C. da Silva et al., Nucl. Instr. and Meth. B 191 (2002) 158.

[3] V. Shuttanandan et al., Applied Physics Letter 84 (2004) 4466.

[4] H.B. Liao et al., Applied Physics Letter 72 (1998) 1817.

[5] This work was supported financially by the NSAF Joint Foundation of China (10376006) and by Program for New Century Excellent Talents in University. 


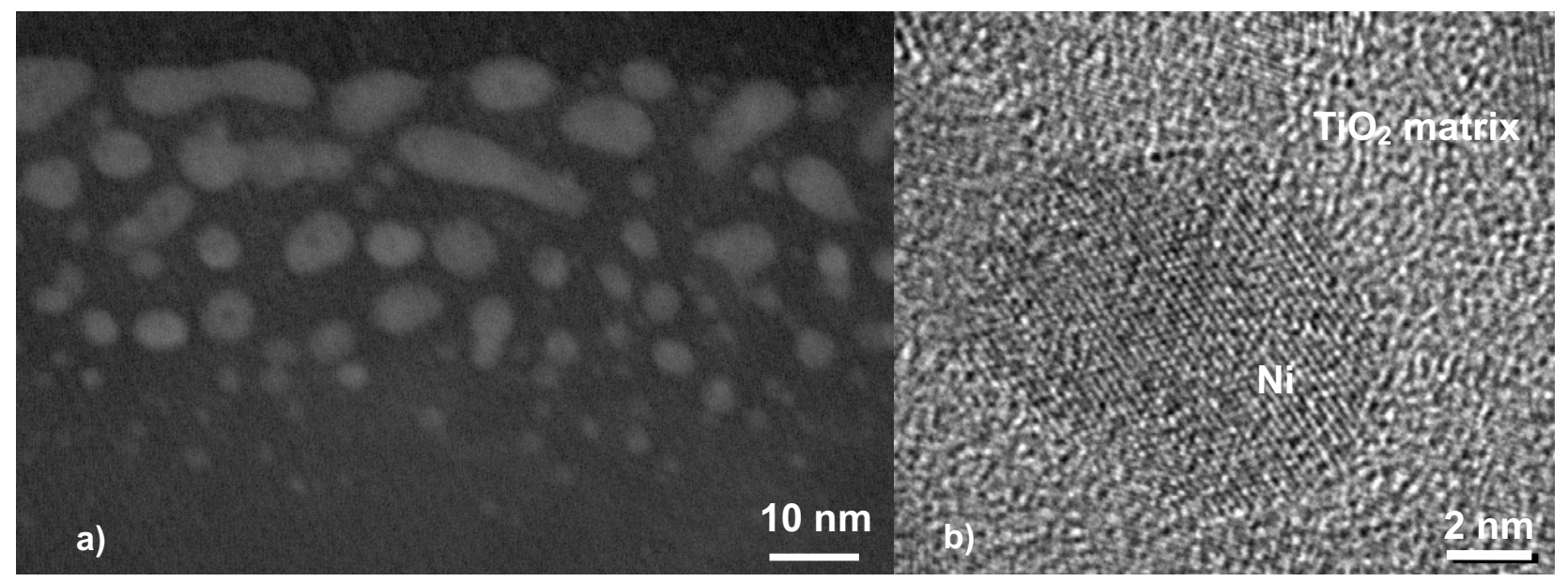

Fig. 1. A Z-contrast STEM image indicating the Ni nanoparticles in $\mathrm{a} \mathrm{TiO}_{2}$ crystal after ion implantation (a); A high resolution TEM micrographs showing crystalline characteristics of Ni nanoparticles in amorphous $\mathrm{TiO}_{2}$ host which result from the implantation damage (b).
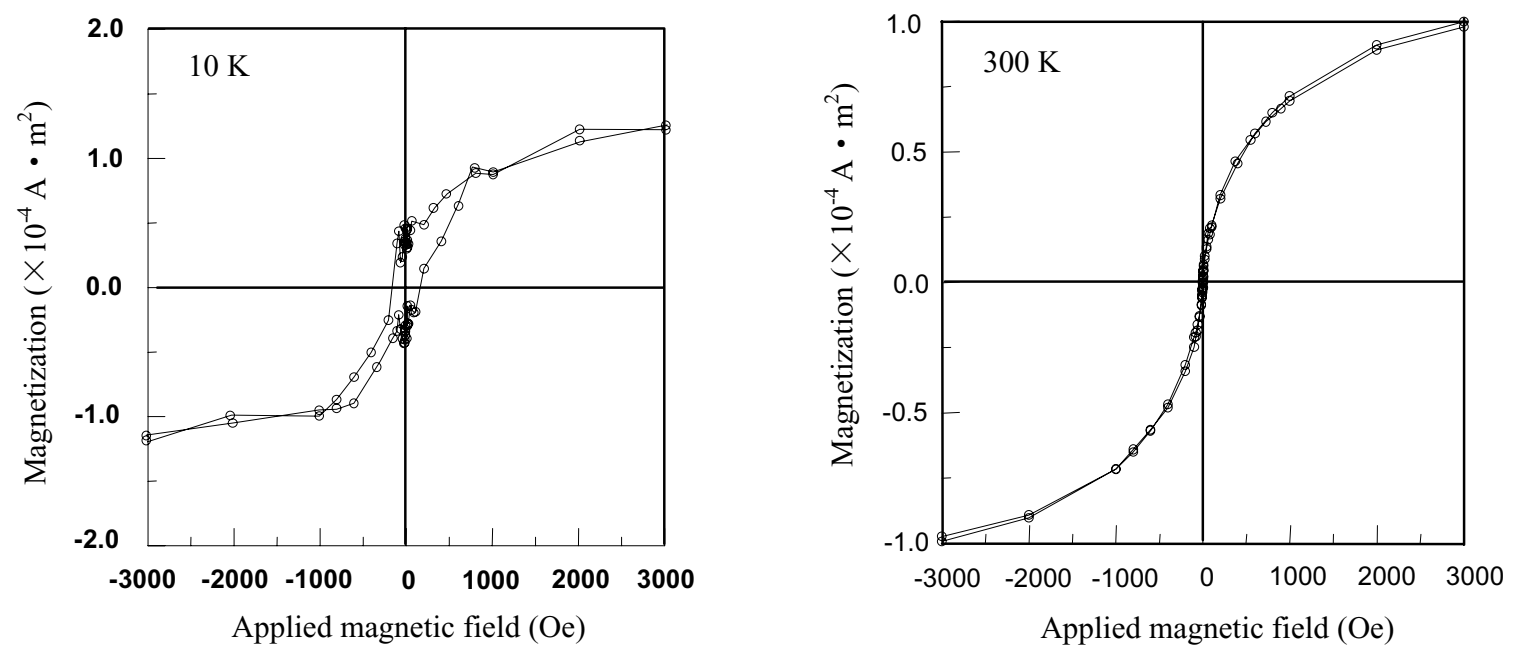

Fig. 2. Magnetic hysteresis loops of Ni nanoparticles in $\mathrm{TiO}_{2}$ single crystals at $10 \mathrm{~K}$ and $300 \mathrm{~K}$ indicating the coercive force of Ni nanoparticles was about $210 \mathrm{Oe}$ at $10 \mathrm{~K}$.

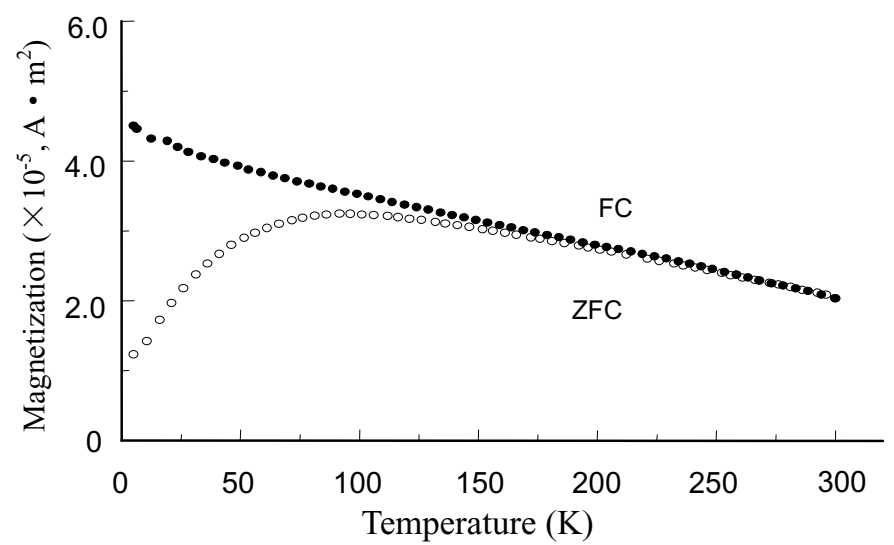

Fig. 3. Temperature dependence of the magnetization showing the $\mathrm{T}_{\mathrm{b}}=85 \mathrm{~K}$. Curves were taken in the $\mathrm{ZFC}$ and $\mathrm{FC}$ processes at $\mathrm{H}=100 \mathrm{Oe}$. 